\title{
Sunitinib, sorafenib and other systemic noncytotoxic kidney cancer therapies can and should be administered by urologists
}

\author{
Laurence Klotz, MD, FRCSC
}

U rology is not just a surgical discipline. More than surgeons from other surgical disciplines, urologists have a long tradition of managing patients with multimodality therapies, including systemic therapies. The most obvious example of this is the use of androgen deprivation therapy for prostate cancer. Other examples include the use of intravesical chemotherapy for bladder cancer, and tumour vaccines and cytokines for renal cancer. Urologists have been at the forefront of research into many systemic therapies for cancer, including androgen-deprivation therapies, anti-androgens, gene therapy for prostate cancer, and atrasentan, zoledronic acid, estramustine, and gamma interferon for kidney cancer. Urologists were involved in the development of systemic chemotherapy for metastatic testicular cancer. For the first 10 years after the introduction of multi-agent chemotherapy for testicular cancer, urologic oncologists administered these drugs in many centres. These physicians acquired clinical skills in the management of patients with metastatic disease and the toxicities associated with these drugs.

Why did this change? Primarily because of drug toxicity. Multi-agent, platinum-based cytotoxic chemotherapies can be lethal to patients. Methotrexate-vinblastineadriamycin-cis-platinum chemotherapy, which was standard therapy for advanced prostate cancer for 20 years, induces fatal neutropenic sepsis in $2 \%-4 \%$ of patients. Physicians administering these regimens need to be highly focused on the morbidities associated with these drugs and the interventions required to manage them.

In this new era of noncytotoxic systemic therapies for cancer, of which the tyrosine-kinase inhibitors are excellent examples, these drugs do have associated toxicities, but their side effects are rarely life-threatening. These drugs do not induce neutropenia; they induce hypertension, handfoot syndrome and other non-life-threatening toxicities. Much like many other agents used in urology, these drugs do require care and experience to administer.

Patients with metastatic cancer are not easy to manage. They experience complications from their malignancies that many urologists may find challenging to treat, for example, the management of malignant ascites, expanding liver metastases or recurrent malignant pleural effusions that cause dyspnea. Some urologists, however, will rise to the challenge and maintain the skills required, in conjunction with palliative care physicians.

The stakes are high. The future of cancer management lies in risk stratification with clinical, biochemical and genetic markers, and multimodality therapy for patients at risk. Chronic-disease management with long-term systemic therapy that uses targeted agents is likely to become common.

In that environment, surgeons may function in 1 of 2 ways. In one model, the medical oncologist acts as the primary caregiver, as is frequently the case for breast and colon cancer in North America. The urologist is relegated to the technical aspects of cancer resection. As many operations currently carried out are replaced by image-guided nonsurgical interventions, this limited role will decline further. The need for urologic oncologists will be limited, the numbers small, and the scope of practice narrow. A future scenario might unfold this way - a family doctor identifies a $4-\mathrm{cm}$ mass during renal ultrasound and refers the patient to a medical oncologist for a management decision. The oncologist sends the patient to an interventional radiologist for biopsy and MRI-guided high-intensity focused ultrasound. The radiologist then sends the patient back to the medical oncologist for chronic adjuvant therapy. Plausible? Definitely. Desirable? You decide.

In the alternative model, urologists function as primary caregivers for patients with urologic cancer, helping the patient make the primary treatment decision, administering neoadjuvant and adjuvant therapy with noncytotoxic therapies, and following the patient through the chronic phase of the disease. Upon progression, relapse or failure of first-line therapy, the urologist would refer patients to a medical oncologist for consultation about further management. This is a much more robust and attractive model of urologic oncology, and in many environments will have benefits for the patient.

One shoe does not fit all, however. This model is appropriate for a subgroup of urologic oncologists with an interest in systemic therapy. It would not be appropriate for general urologists with limited interest and experience in managing patients with advanced cancer. Many factors will have an impact, including workload, resource base, and 
location (rural or urban) and type (academic or community) of practice. The interest and experience of the treating physician will be key. The availability and collaboration of medical oncologists with an interest in genitourinary cancer may obviate the benefit of initiatives in this area.

Urologists embarking on this path must obtain training in the use of agents for systemic therapy and structure their practice so that it can accommodate the increased needs of patients with advanced cancer. These urologists should seek a close working relationship with a medical oncologist and pursue a multidisciplinary model of care. However, the multidisciplinary model should not come at the expense of losing our hallowed status as primary care physicians for patients with urologic cancer, including renal cancer.

From the Department of Surgery, University of Toronto and the Division of Urology, Sunnybrook Health Sciences Centre, Toronto, Ont.

This article has been peer reviewed.

Competing interests: None declared.

Correspondence: Dr. Laurence Klotz, Sunnybrook Health Sciences Centre, 2075 Bayview Ave. \#MG408, Toronto ON M4N 3M5; Laurence.Klotz@sunnybrook.ca

\title{
A medical oncologist is the most qualified specialist to provide systemic therapy for patients with advanced renal cell carcinoma
}

\author{
Peter Venner, $M D$
}

I t has been suggested that urologists, like medical oncologists, could be responsible for administering the systemic therapy required by patients with advanced renal cell carcinoma (RCC). This debate seems to have arisen because the new agents coming onto the market are oral, rather than the more traditional intravenous, agents. As a consequence, physicians may think that these new agents are simple to administer, require limited monitoring and, therefore, less expertise than conventional chemotherapy.

In the early days of chemotherapy, various physicians who had an interest in managing patients with advanced cancer administered these drugs. However, in the 1970s in the United States and the 1980s in Canada, treatment regimens became more effective and complicated. Medical oncology, which evolved from clinical hematology in North America and radiation oncology in Europe, became a recognized subspecialty of internal medicine with well-defined training requirements and a clearly designated scope of practice. As a consequence, patients receiving chemotherapy should be cared for by internists with these qualifications. The wide range of drugs often administered within complex protocols that may be associated with life-threatening toxicities have removed the care of these patients from unqualified practitioners dabbling in chemotherapy because they want to or have done so in past. In addition, the specialist has to recognize the indications for chemotherapy, along with knowing when it should be set aside in favour of other modalities of care, including surgery, radiation therapy, supportive care or palliative care.

Traditionally, urologists have managed some patients on systemic therapy, usually with agents that are modifications of common surgical procedures, such as chemical castration for advanced prostate cancer. In addition, some urologists have supervised the care of patients on interferon. Because this agent is less likely to have the serious or lifethreatening side effects commonly associated with chemotherapy, these patients can be supervised by physicians with less experience or training than that required for patients on chemotherapy.

However, extending this perception to the newly available agents for the systemic therapy of patients with advanced RCC is a serious misconception. It would be foolhardy for unqualified physicians to undertake the management of these potentially very complicated patients. Make no mistake, the new agents for advanced RCC are chemotherapy drugs and must be afforded appropriate respect for their toxicities and acknowledgement of their effectiveness. Patients on these drugs need the expert care of appropriately trained physicians working with an experienced healthcare team.

I believe that primarily one's training, followed by experience should determine one's scope of practice. In Great Britain, physicians are asked when they qualified, a term used to identify when they finished their training and 
attained the qualifications that allow them to practice as specialists. To determine what these qualifications are one needs to examine the requirements and objectives of the training programs from which they graduated, which also defines their scope of practice and the privileges they will be given within a hospital or ambulatory care setting. In addition, agencies responsible for providing cancer drugs often restrict their prescribing to those who are qualified to administer these agents.

The requirements of graduates of accredited Canadian specialty programs that relate to this debate on systemic therapy are described on the Web site of the Royal College of Physicians and Surgeons for both urology and medical oncology in the relevant sections of which are cited below.

Urology:

For all tumours (benign and malignant) of the genito-urinary tract, residents will be able to describe the etiology, prevention, nutritional and environmental aspects of urologic malignant disease, natural history, histology and pathology, investigation and diagnostic techniques, grading and staging techniques in common use, principles of cancer management, role of surgery, radiotherapy (external beam and brachytherapy), chemotherapy, immunotherapy, angioinfarction and cryotherapy for each stage and the principles of management when cure is not the primary goal. ${ }^{1}$

Although these are very laudable goals, nowhere does this description of requirements state that a urology resident and ultimately a urologist should be trained to administer chemotherapy or manage patients who are on chemotherapy. Today, urology graduates must know the role of tyrosine-kinase inhibitors and other agents in the management of advanced RCC, but they do not need to know the specific details of how to administer the drugs or manage patients on these agents. An infantryman does not need to know how to lay down a cannon barrage (but he should no when to call one in).

The medical oncologist's training in the care of patients with cancer and the administration of chemotherapy provides a decided contrast.

Some of the specific aspects of knowledge with which the resident should become familiar include the following:

3. indications for and complications of the various treatment modalities alone or in combination, together with an appreciation of their curative or palliative potential;

4. management of medical emergencies and complications which may arise as a result of cancer or its treatment and palliative symptomatic treatment;

5. basic scientific knowledge including growth kinetics, molecular biology, biochemistry, pharmacology, endocrinology and im- munology as they relate to the understanding of cancer and its treatment. ${ }^{2}$

These contrasting descriptions make it clear that the training of urologists has a very different focus than that of medical oncologists. Urology is a surgical specialty, whereas medical oncology is based on systemic therapy. Urologists have training that allows them to develop the expertise to deal directly with the surgical aspects of cancer and to understand the principles of cancer management, including the role of chemotherapy without hands-on experience of it. On the other hand, medical oncologists have to be able to provide advice and care, including systemic therapy, to patients with more advanced disease. As is evident from the requirements of these training programs, physicians graduating from urology programs are no more trained to manage patients on chemotherapy than a medical oncologist is trained to do cystoscopies or nephrectomies. It should go without saying that specialists need to recognize their area of expertise and those of others.

What is it, then, that medical oncologists are trained to do to care for their patients with cancer, particularly when this care involves chemotherapy? First of all, based on their knowledge of these patients, their cancer and the available therapies, medical oncologists can counsel patients about whether systemic therapy is advisable and what to expect of it. Medical oncologists are also knowledgeable about the natural history of patients with cancer and are able to advise patients about other therapeutic options such as metastasectomy for limited disease or the best supportive care for patients with an extremely limited chance of response or survival, based on recognized prognostic factors. If the decision is made to proceed with chemotherapy, it is the medical oncologist's responsibility to provide ongoing care, and to manage the treatment and any complications that arise in association with it.

The complications or common side effects that medical oncologists manage daily, and urologists rarely deal with, include the consequences of bone-marrow suppression, emesis, anorexia, fatigue, mucositis, and dysgeusia, and less commonly, skin toxicity, hypertension, abnormal results of renal, liver and pancreatic function tests, thyroid suppression, and impaired cardiac output, among others. In addition, the medical oncologist has to assess evolving symptoms and be able to distinguish those arising from chemotherapy from those caused by the cancer. In the instances in which the chemotherapy is not achieving its intended goals, the medical oncologist has to recognize this and advise patients of alternatives to the current therapy.

Medical oncologists have the expertise to evaluate a patient and advise about the risks and benefits of systemic therapy. 
This expertise is garnered not only from their training, but also from the administration of chemotherapy to the hundreds of patients whom they will encounter in their practice.

Although the toxicity profile of tyrosine-kinase inhibitors has been reported in recent publications, it is worthwhile to review them here - if only to put fear into the hearts of the naïve, yet adventuresome soul. Table 1 summarizes the overall frequency of the most common side effects and more serious problems encountered by patients treated with sunitinib, as reported in the paper demonstrating its superiority over interferon as first-line therapy for patients with metastatic RCC.

These are not trivial problems; on occasion they are lifethreatening and require a clinician with sufficient training and experience to safely manage patients on these drugs. Familiarity with other chemotherapy agents and protocols provides physicians and healthcare teams with the knowledge they need to deal with these problems. A clinician would be on shaky ground if he or she were not able to recognize the seriousness of the problems and make appropriate, timely decisions to manage these side effects. Already faced with a serious, lifespan-shortening disease, patients need to have confidence that the person responsible for their care is qualified to do so. If a member of my family had RCC, I am not sure that I would have confidence in my urological colleagues' ability to provide the required care and support. As well, I would think that, as part of wise risk management, a specialist would be well advised to entrust patient care to colleagues with this exper-

\section{Table 1. A summary of the frequency of clinical and laboratory side effects reported in a phase 3 study of sunitinib in metastatic $\mathbf{R C C}^{3}$}

\begin{tabular}{lcc}
\hline & \multicolumn{2}{c}{ Frequency, \% } \\
\hline Adverse event & All grades & Grade 3 or 4 \\
\hline Diarrhea & 53 & 5 \\
Fatigue & 51 & 7 \\
Nausea & 44 & 3 \\
Stomatitis & 25 & 1 \\
Vomiting & 24 & 4 \\
Hypertension & 24 & 8 \\
Hand-foot syndrome & 20 & 5 \\
Mucosal inflammation & 20 & 2 \\
Rash & 19 & 1 \\
Asthenia & 17 & 4 \\
Leucopenia & 78 & 5 \\
Neutropenia & 72 & 12 \\
Anemia & 71 & 4 \\
Thrombocytopenia & 65 & 8 \\
Increased lipase/amylase & $52 / 32$ & $16 / 5$ \\
Increased creatinine & 66 & 1 \\
Liver function test elevation & $46-52$ & $2-3$ \\
\hline RcC = renal cell carcinoma. & & \\
\hline
\end{tabular}

tise. When this expertise is readily available, why would one want to have to explain to patients or their families, licensing bodies and insurance companies about a misadventure during the administration of chemotherapy? It is difficult enough to do this when it happens in the most experienced of hands. In addition, oncologists have to have the confidence and experience to know when to consult with their surgical or radiation oncology colleagues without fearing that their patient will be lost to their practice.

That said, medical oncologists do not work in isolation. The management and care of patients with RCC requires the expertise of a multidisciplinary team. Patients have disease burdens that vary from asymptomatic renal lesions to overwhelming metastatic disease. They may have one of a myriad of paraneoplastic syndromes, in addition to a host of comorbid conditions. For these patients to have appropriate, safe and state-of-the-art care, their physicians must have the right training, qualifications, experience and expertise. In addition, this care must be provided in an environment, ideally in a cancer centre, where patients will be monitored and supported by other members of the healthcare team who are experienced in providing chemotherapy. Being cared for in this environment helps to ensure the availability of appropriate backup if problems arise after regular hours or when regular care providers are unavailable.

Finally, tyrosine-kinase inhibitors are not the only drugs available, or soon to be available, for the management of patients with advanced RCC. Some patients may still be eligible for cytokine therapy; others may be appropriately treated with mammalian target of rapamycin inhibitors. How and when to incorporate these other agents will require the expertise of a medical oncologist.

I would be remiss if I did not state that a few urologists have taken the time to learn how to administer some systemic agents safely and appropriately. If others want to learn these skills and develop the expertise required to manage patients with advanced RCC, they are welcome to do so. Unfortunately, there is no shortage of work for medical oncologists (as may not be the case for urologists in some centres), and we are always willing to explore opportunities to use the assistance of others who can provide care in a safe and appropriate manner. If urologists find themselves with the time for and the commitment to learning new skills, I am sure my colleagues could provide them with the expertise they need so that they could have a meaningful role on the healthcare team responsible for the care of patients with advanced RCC.

In summary, the new chemotherapeutic agents coming onto the market for the management of patients with advanced RCC fall into the purview of medical 
oncologists. They have the training, expertise and temperament to care for these potentially very sick patients. Because complete responses to therapy are not likely, this disease may become chronic, requiring the long-term involvement and support of the caregiver, a quality commonly seen in medical oncologists whose medical subspecialty is no stranger to the care of patients with chronic diseases. Patients will be better served, as will the health care system, by having their care provided by a specialist trained in the administration of chemotherapy and familiar with the demands of caring for patients with incurable, but very treatable cancer. RCC remains an internist cancer, and advanced RCC, even more so. Because of its complexity, patients with advanced RCC should be cared for by physicians from a cognitive specialty that has been augmented by further subspecialty training and experience. These physicians are medical oncologists, not urologists.

\section{References}

1. Information by specialty or subspecialty (Urology). [Web site of the Royal College of Physicians and Surgeons]. Available: http://rcpsc.medical.org/information/index.php? specialty=360\&submit=Select (accessed 2007 May 8).

2. Information by specialty or subspecialty (Medical oncology). Web site of the Royal College of Physicians and Surgeons]. Available: http://rcpsc.medical.org/information/index.php?specialty=452\&submit $=$ Select (accessed 2007 May 8).

3. Motzer RJ, Hutson TE, Tomczak P, et al. Sunitinib versus interferon alfa in metastatic renal-cell carcinoma. N Engl J Med 2007;356:115-24

From the Department of Medical Oncology, Cross Cancer Institute and Division of Medical Oncology, Department of Oncology, University of Alberta, Edmonton, Alta.

This article has been peer reviewed.

Competing interests: None declared.

Correspondence: Dr. Peter Venner, Cross Cancer Institute and the Department of Oncology, University of Alberta, 11560 University Ave., Edmonton AB T6G 122; peterven@cancerboard.ab.ca 